\title{
Molecular characterization and PCR-based replicon typing of multidrug resistant Shigella sonnei isolates from an outbreak in Thimphu, Bhutan
}

Sirigade Ruekit ${ }^{*}$, Sonam Wangchuk ${ }^{2}$, Tshering Dorji ${ }^{2}$, Kinzang Pem Tshering ${ }^{3}$, Piyarat Pootong ${ }^{1}$, Panida Nobthai ${ }^{1}$, Oralak Serichantalergs ${ }^{1}$, Kamonporn Poramathikul', Ladaporn Bodhidatta ${ }^{1}$ and Carl Jeffries Mason ${ }^{1}$

\begin{abstract}
Background: Shigella species are an important cause of diarrhea in developing countries. These bacteria normally acquire their antibiotic resistance via several different mobile genetic elements including plasmids, transposons, and integrons involving gene cassettes. During a diarrhea surveillance study in Thimphu, Bhutan in June and July, 2011, Shigella sonnei were isolated more frequently than expected. This study describes the antibiotic resistance of these S. sonnei isolates.
\end{abstract}

Methods: A total of 29 S. sonnei isolates from Thimphu, Bhutan was characterized for antimicrobial susceptibility by disc diffusion assay and minimum inhibitory concentration (MIC) assay. All isolates were tested by pulsed-field gel electrophoresis (PFGE) with restriction enzyme Xbal and were tested for plasmid. The plasmid patterns and the PFGE patterns were analyzed by Bionumerics software. DNA sequencing was performed on amplified products for gyraseA gene and class 1 and class 2 integrons. S. sonnei isolates were classified for incompatibility of plasmids by PCR-based replicon typing (PBRT).

Results: These S. sonnei were resistant to multiple drugs like ciprofloxacin, nalidixic acid, trimethoprim-sulfamethoxazole, streptomycin, and tetracycline but susceptible to azithromycin. All isolates had class 2 integrons dfrA1, sat1 and aadA1 genes. Two point mutations in Gyrase A subunit at position Ser83Leu and Asp87Gly were detected in these quinolone resistant isolates. The plasmid and PFGE patterns of $S$. sonnei isolates suggested a clonal relationship of the isolates. All isolates carried common ColE plasmid. ColE plasmid co-resided with B/O plasmid (nine isolates) or 11 plasmid (one isolate).

Conclusions: The characteristics of 29 S. sonnei isolates from Thimphu, Bhutan in June and July, 2011 are identical in PFGE, plasmid and resistance pattern. This study suggests that these recent S. sonnei isolates are clonally related and multidrug-resistant.

Keywords: Shigella sonnei, Multidrug resistance, Bhutan

\section{Background}

Shigella species are an important cause of diarrhea in developing countries. In 2013, the World Health Organization (WHO) reported the diarrheal disease is a leading cause of child mortality and morbidity in the world, and mostly results from contaminated food and water sources. Worldwide, 780 million individuals

\footnotetext{
*Correspondence: sirigader@afrims.org

${ }^{1}$ Department of Enteric Diseases, Armed Forces Research Institute of Medical Sciences (AFRIMS), Bangkok, Thailand

Full list of author information is available at the end of the article
}

lack access to improved drinking-water and 2.5 billion lack improved sanitation. Diarrhea due to infection is widespread throughout developing countries. Globally, there are nearly 1.7 billion cases of diarrheal disease every year. Each year diarrhea kills around 760, 000 children under five years of age [1]. Bhutan is a developing country in the South Asia region with decent health care access throughout the country. The current population of the country is around 706,800 with about $80 \%$ of the population residing in rural areas. Children under 5 years of age comprise about $10 \%$ of the total population

\section{() Biomed Central}


with an annual birth cohort of 14,000 [2]. For Bhutan, diarrheal disease is an important public health problem. Treatment for diarrheal disease in the country is empirical rather than evidence based due to the lack of diarrhea etiology data. In the Annual Health Bulletin, Bhutan reported an average of 60,000-70,000 cases of diarrhea and 25,000-30,000 cases of dysentery per year from 2005-2009. The overall incidence of diarrheal diseases in Bhutan during 2010 was 126.6 per 1,000 people. The percentage of diarrhea and dysentery that occurred in children less than 5 years of age for 2009 were 34\% and $30 \%$, respectively [3]. Shigellosis has been a notifiable disease in Bhutan since May 2010 with only one case reported [4]. In 2011, a reported diarrhea outbreak probably caused by Shigella flexneri occurred during March 2011 in a remote village in Mongar District, Bhutan [5]. During a diarrhea surveillance study in Thimphu, Bhutan from March 2011 to February 2013, Shigella sonnei was isolated from children's stool samples more frequently than expected in June and July 2011.

The rapid emergence of multidrug-resistant (MDR) strains is largely due to their ability to acquire and disseminate exogenous genes associated with mobile genetic elements such as, transposons, integrons, plasmids, and genomic islands [6]. Some transposons code for single resistance [e.g. TEM b-lactamase (Tn3), kanamycin $(\operatorname{Tn} 5)$ and tetracycline resistance $(\operatorname{Tn} 10)]$, but plasmids and transposons coding multiple drug resistance often possess another genetic element, the integron [7]. Integrons are genetic elements that contain the components of a site-specific recombination system that recognizes and captures mobile gene cassettes. Based on the characteristics of their integrase genes, four classes of integrons (classes 1, 2, 3, and 4) have been identified to date [8]. Class 1 and 2 integrons have been frequently reported in studies of human Shigella [9]. Antibiotic-resistance plasmids frequently contain genes conferring resistance to several different antibiotics. The ability to recognize and categorize plasmids in homogeneous groups on the basis of their phylogenetic relatedness can be helpful to analyze their distribution in nature, the relationship with the host cell and to discover their evolutionary origin [10].

To further study the multidrug resistance in Thimphu, Bhutan, we characterized S. sonnei isolates from children, the genetic basis of antibiotic resistance including class 1 and class 2 integrons, DNA gyrase A mutations and plasmid replicon typing.

\section{Methods}

\section{Clinical specimens}

A diarrhea etiology study was conducted at Jigme Dorji Wangchuk National Referral Hospital (JDWNRH) in Thimphu, Bhutan from March 2011 to February 2013.
Children 3 months to 5 years of age presenting for clinical care at JDWNRH with a chief complaint of acute diarrhea ( 3 or more loose stools in the previous 24 hours starting no more than 72 hours before presenting were eligible to provide a stool sample as cases. Children 3 months to 5 years presenting for clinical care at JDWNRH for other causes, who not had a history of diarrhea in the past 2 weeks and had not received antibiotics in the past 3 days were eligible to provide stool samples as controls. The study was approved by the Research Ethics Board of Health (REBH), Bhutan and the Institutional Review Board (IRB), Walter Reed Army Institute of Research (WRAIR). Stool samples were collected from children with verbal informed consent from their parent or guardian.

\section{Specimen processing and identification}

Stool samples collected in Cary-Blair transport medium were cultured on MacConkey (MAC) and Hektoen Enteric (HE) agar (Becton-Dickinson and Company, Sparks, MD, USA) and subsequently incubated at $37^{\circ} \mathrm{C}$ overnight. Plates are examined for the presence of colonies resemble to Shigella and identified by standard biochemical tests and further serotyped by agglutination test (Denka Seiken, Japan) at the Armed Forces Research Institute of Medical Sciences (AFRIMS) in Bangkok, Thailand [11]. Isolates were stored in Luria-Bertani (LB) broth containing $15 \%$ glycerol at $-70^{\circ} \mathrm{C}$ until use.

\section{Antimicrobial susceptibility methods}

The S. sonnei isolates were tested for susceptibility to antimicrobial agents by the standard disk diffusion method according to Clinical and Laboratory Standards Institute (CLSI) guideline [12]. Antimicrobial disks included azithromycin (AZM $15 \mu \mathrm{g}$ ), nalidixic acid (NA $30 \mu \mathrm{g}$ ), ciprofloxacin (CIP $5 \mu \mathrm{g}$ ), ampicillin (AM $10 \mu \mathrm{g}$ ), trimethoprim-sulfamethoxazole (SXT 1.25/23.75 $\mu \mathrm{g}$ ), ceftriaxone (CRO $30 \mu \mathrm{g})$, streptomycin (S $10 \mu \mathrm{g})$, and tetracycline (TE $30 \mu \mathrm{g})$ (Sensi-Disc; Becton Dickinson, NJ, USA). Minimum inhibitory concentrations (MIC) for AZM, NA, and CIP were also determined by E-test (AB Biodisk, Solna, Sweden). The standard disk diffusion method and MIC were interpreted according to CLSI guideline [12]. Organisms used for quality control of antimicrobial susceptibility testing included Escherichia coli ATCC 25922, Staphylococcus aureus ATCC 25923, and Staphylococcus aureus ATCC 29213. In the absence of CLSI definitive standards for azithromycin (AZM) breakpoints of the family Enterobacteriaceae, interpretive standards as published by the disk manufacturer for Staphylococcus spp. was used (BBL package insert; Becton-Dickinson and Company, Sparks, MD, USA). Multidrug-resistant (MDR) S. sonnei strains were defined as strains that were resistant to two or more antimicrobial agents. 


\section{Pulsed-field gel electrophoresis (PFGE)}

Pulsed-field gel electrophoresis (PFGE) was performed according to the One-Day (24-28 h) Standardized Laboratory Protocol for Molecular Subtyping by PFGE [13] with minor modifications. The cell density of each isolate was adjusted by spectrophotometer (Bio-Rad, Hercules, CA, USA). Plug slices of samples and Salmonella Braenderup H9812 universal size standard were prepared for XbaI digestion and loaded on 1\% SeaKem Gold agarose (Lonza, Rockland, ME, USA) with 0.5x TBE pH 8.3 ( $890 \mathrm{mM}$ Tris, $890 \mathrm{mM}$ Borate, $20 \mathrm{mM}$ EDTA). The gel was electrophoresed by CHEF Mapper system (Bio-Rad, Hercules, CA, USA). The electrophoresis conditions for $\mathrm{X} b a \mathrm{I}$ digestion were auto algorithm mode, 30-600 kb for molecular weight range, and $19 \mathrm{~h}$ for run time. The gel images were captured by a gel documentation system (Syngene, Cambridge, UK). Combined dendrogram of PFGE and antimicrobial susceptibility were generated by BioNumerics Software version 6.0 (Applied Maths, Kortrijk, Belgium), UPGMA (Unweighted Pair Group Method with Arithmetic Mean) type and Dice coefficient with $1.0 \%$ optimization and tolerance.

\section{Plasmid analysis}

The S. sonnei isolates, E. coli V.517 and E. coli 39R were grown in $5 \mathrm{ml} \mathrm{LB}$ broth at $200 \mathrm{rpm}, 37^{\circ} \mathrm{C}$ overnight. Plasmid DNAs were extracted by alkaline lysis method [14] and separated by electrophoresis on a $0.8 \%$ agarose gel with $0.5 \mathrm{x}$ TBE using plasmids from E. coli V.517 and E. coli $39 \mathrm{R}$ as reference size markers. A combined dendrogram of plasmid and antimicrobial susceptibility were generated by BioNumerics Software version 6.0 (Applied Maths, Kortrijk, Belgium), UPGMA type and Dice coefficient with $1.5 \%$ optimization and tolerance.

\section{Multiplex PCR for plasmid replicon typing}

The $S$. sonnei isolates were examined by PCR using three multiplex primer panels for the presence of 18 replicon plasmids and three singleplex primer pairs for an additional 3 replicon plasmids, ColE, IncU and IncR plasmids (Table 1). S. sonnei isolates and reference plasmids for controls were grown on TSA plates at $37^{\circ} \mathrm{C}$ overnight and were extracted for genomic DNA using DNeasy Blood and Tissue Kit (QIAGEN, Valencia, CA, USA). The reference plasmid controls were kindly provided by Dr. Alessandra Carottoli. PCR reactions were performed as previously described $[15,16]$. Positive controls as well as a negative control without DNA were run with each multiplex primer panel.

\section{Amplification of Integrons, DNA gyrase A gene and azithromycin resistance gene ( $m p h A)$}

The S. sonnei isolates were grown on TSA plates at $37^{\circ} \mathrm{C}$ overnight and purified for genomic DNA extracted using
DNeasy Blood and Tissue Kit (QIAGEN, Valencia, CA, USA). DNA templates were kept at $-20^{\circ} \mathrm{C}$ before use. Extracted DNA was amplified for class 1 and class 2 integrons, DNA gyrase A gene and azithromycin resistance gene $(m p h A)$ by PCR (Table 1$)$, as previously described [17-19].

\section{Restriction fragment length polymorphism (RFLP)}

RFLP was performed on amplified DNA of class 2 integrons of the S. sonnei isolates by using Int $2 \mathrm{CaF}$ and Int $2 \mathrm{CaR}$ primers (Table 1) and PCR conditions as previously described [17]. Amplified product was purified by Wizard ${ }^{\circ}$ SV Gel and PCR Clean-Up System (Promega, Madison, WI, USA) and digested with $\mathrm{XbaI}$ (Roche, Indianapolis, IN, USA) at $37^{\circ} \mathrm{C}$ for 2 hours. The restriction fragments were detected on a $1.5 \%$ agarose gel by electrophoresis with $0.5 \mathrm{X}$ TBE buffer. Two-log DNA ladder (BioLabs, Ipswich, MA, USA) was used as a standard DNA marker.

\section{Sequencing and bioinformatics analysis}

The amplified PCR products of S. sonnei isolates, class 1 integrons, class 2 integrons and the gyrase A gene were purified by Wizard ${ }^{\circ} \mathrm{SV}$ Gel and PCR Clean-Up System and were sequenced (Macrogen, Korea). The nucleotide sequences were analyzed with BLAST software (www. ncbi.nlm.nih.gov/BLAST).

\section{Results}

\section{Shigella sonnei isolates}

During the surveillance study from March 2011 to February 2013 at JDWNRH in Thimphu, Bhutan, a total of 1,133 children from 3 months to 5 years of age were studied. This included 571 children with diarrhea and 562 non-diarrhea controls. The percentage of Shigella spp. detected were $70.9 \%(56 / 79)$ of S. sonnei, 19.0\% (15/79) of S. flexneri, 8.9\% (7/79) of S. boydii and 1.2\% (1/79) of $S$. dysenteriae. An epidemic curve of the number of cases per month of S. sonnei from March 2011 to February 2013 was created, a total of $56 \mathrm{~S}$. sonnei isolates were 51 isolates from cases and 5 isolates from controls (Figure 1). During the apparent outbreak of S. sonnei in Thimphu, Bhutan in June and July 2011, a total of $25 \mathrm{~S}$. sonnei were isolated from cases and $4 S$. sonnei were isolated from asymptomatic controls. The other $27 \mathrm{~S}$. sonnei isolates were sporadically found from March 2011 to February 2013. All 29 S. sonnei isolates during the outbreak period were characterized in this study. Four S. sonnei from Nepal in 2007-2008 and five S. sonnei from Thailand in 2008 isolated previously at AFRIMS were included in this study for comparison.

\section{Multidrug-resistant Shigella sonnei in Bhutan}

In this study, the $29 \mathrm{~S}$. sonnei isolates from Thimphu, Bhutan were resistant to NA, CIP, SXT, S and TE by disk diffusion assay (Table 2). These 29 isolates had 
Table 1 PCR primer sequence

\begin{tabular}{|c|c|c|c|c|c|}
\hline Primer & Sequence $\left(5^{\prime}-3^{\prime}\right)$ & Location & Annealing temperature $\left({ }^{\circ} \mathrm{C}\right)$ & $\begin{array}{c}\text { Product } \\
\text { size (bp) }\end{array}$ & Reference \\
\hline IntF & GGCATCCAAGCAGCAAGC & $5^{\prime}-\mathrm{CS}$ of class 1 integrons & 58 & varied & {$[17]$} \\
\hline $\ln B$ & AAGCAGACTTGACCTGAT & $3^{\prime}$-CS of class 1 integrons & & & \\
\hline Int12L & GTAGCAAACGAGTGACGAAATG & intl2 & 58 & 789 & {$[17]$} \\
\hline $\ln t \mid 2 R$ & CACGGATATGCGACAAAAAGGT & & & & \\
\hline Intl2CaF & GATAAAAACAGCCTGACCTCTTC & intl2 & 58 & 2433 & [17] \\
\hline Intl2CaR & CCCACTTGACATCTCATCAATAC & $3^{\prime}$ region of class 2 integrons & & & \\
\hline ST.GYRA1 & TGTCCGAGATGGCCTGAAGC & DNA gyrase A & 55 & 470 & [18] \\
\hline ST.GYRA12 & CGTTGATGACTTCCGTCAG & & & & \\
\hline mphAF & AACTGTACGCACTTGC & macrolide 2'-phosphotransferase & 50 & 837 & [19] \\
\hline mphAR & GGTACTCTTCGTTACC & & & & \\
\hline \multicolumn{6}{|l|}{ Multiplex 1} \\
\hline $\mathrm{B} / \mathrm{O}_{-} \mathrm{F}$ & GCGGTCCGGAAAGCCAGAAAAC & RNAI & 60 & 159 & [15] \\
\hline$B / O \_R$ & TCTGCGTTCCGCCAAGTTCGA & & & & \\
\hline FIC_F & GTGAACTGGCAGATGAGGAAGG & repA2 & 60 & 262 & {$[15]$} \\
\hline FIC_R & TTCTCCTCGTCGCCAAACTAGAT & & & & \\
\hline$A / C_{-} F$ & GAGAACCAAAGACAAAGACCTGGA & repA & 60 & 465 & [15] \\
\hline$A / C \_R$ & ACGACAAACCTGAATTGCCTCCTT & & & & \\
\hline$P_{-} F$ & CTATGGCCCTGCAAACGCGCCAGAAA & iterons & 60 & 534 & [15] \\
\hline$P \_R$ & TCACGCGCCAGGGCGCAGCC & & & & \\
\hline T_F & TTGGCCTGTTTGTGCCTAAACCAT & repA & 60 & 750 & [15] \\
\hline T_R & CGTTGATTACACTTAGCTTTGGAC & & & & \\
\hline \multicolumn{6}{|c|}{ Multiplex 2} \\
\hline KB_F & GCGGTCCGGAAAGCCAGAAAAC & RNAI & 60 & 160 & [15] \\
\hline KVB_R & TCTITCACGAGCCCGCCAAA & & & & \\
\hline W_F & CCTAAGAACAACAAAGCCCCCG & repA & 60 & 242 & [15] \\
\hline W_R & GGTGCGCGGCATAGAACCGT & & & & \\
\hline FIIA_F & CTGTCGTAAGCTGATGGC & repA & 60 & 270 & {$[15]$} \\
\hline FllA_R & CTCTGCCACAAACTTCAGC & & & & \\
\hline FIA_F & CCATGCTGGTTCTAGAGAAGGTG & iterons & 60 & 462 & [15] \\
\hline FIA_R & GTATATCCTTACTGGCTTCCGCAG & & & & \\
\hline FIB_F & GGAGTTCTGACACACGATTTTCTG & repA & 60 & 702 & [15] \\
\hline FIB_R & CTCCCGTCGCTTCAGGGCATT & & & & \\
\hline Y_F & AATTCAAACAACACTGTGCAGCCTG & repA & 60 & 765 & [15] \\
\hline Y_R & GCGAGAATGGACGATTACAAAACTTT & & & & \\
\hline \multicolumn{6}{|c|}{ Multiplex 3} \\
\hline 11_F & CGAAAGCCGGACGGCAGAA & RNAI & 60 & 139 & [15] \\
\hline I1_R & TCGTCGTTCCGCCAAGTTCGT & & & & \\
\hline Frep_F & TGATCGTTAAAGGAATTTTG & $\mathrm{RNAl} / \mathrm{repA}$ & 60 & 270 & [15] \\
\hline Frep_R & GAAGATCAGTCACACCATCC & & & & \\
\hline X_F & AACCTTAGAGGCTATTTAAGTTGCTGAT & ori $\gamma$ & 60 & 376 & {$[15]$} \\
\hline$X \_R$ & TGAGAGTCAATIITIATCTCATGTITAAGC & & & & \\
\hline HI1_F & GGAGCGATGGATTACTTCAGTAC & parA-parB & 60 & 471 & [15] \\
\hline HI1_R & TGCCGTTTCACCTCGTGAGTA & & & & \\
\hline
\end{tabular}


Table 1 PCR primer sequence (Continued)

\begin{tabular}{|c|c|c|c|c|c|}
\hline N_F & GTCTAACGAGCTTACCGAAG & repA & 60 & 559 & [15] \\
\hline N_R & GTTTCAACTCTGCCAAGTTC & & & & \\
\hline $\mathrm{HI} 2 \mathrm{~F}$ & TTCTCCTGAGTCACCTGTTAACAC & iterons & 60 & 644 & {$[15]$} \\
\hline $\mathrm{H} 12 \mathrm{R}$ & GGCTCACTACCGTTGTCATCCT & & & & \\
\hline L/M_F & GGATGAAAACTATCAGCATCTGAAG & repA, B, C & 60 & 785 & [15] \\
\hline L/M_R & CTGCAGGGGCGATTCTTTAGG & & & & \\
\hline oricolE_F & GTTCGTGCATACAGTCCA & ColE plasmids & 60 & 187 & [16] \\
\hline oricolE_R & GGCGAAACCCGACAGGAC & & & & \\
\hline IncR_F & TCGCTTCATTCCTGCTTCAGC & IncR plasmids & 60 & 251 & {$[16]$} \\
\hline IncR_R & GTGTGCTGTGGTTATGCCTCA & & & & \\
\hline IncU_F & TCACGACACAAGCGCAAGGG & IncU plasmids & 60 & 843 & [16] \\
\hline IncU_R & TCATGGTACATCTGGGCGC & & & & \\
\hline
\end{tabular}

an MIC of NA $(>256 \mu \mathrm{g} / \mathrm{ml})$ and CIP $(3.0-4.0 \mu \mathrm{g} / \mathrm{ml})$ by E-test confirming the resistance described by disk diffusion assay. All 29 isolates were susceptible to AZM by disk diffusion assay. The MIC range of AZM by E-test was $3.0-4.0 \mu \mathrm{g} / \mathrm{ml}$ corresponding to intermediate susceptibility to AZM (Table 2).

\section{PFGE patterns and plasmid patterns analysis}

At $80 \%$ similarity, the PFGE patterns of all 38 isolates clustered into 4 groups (Figure 2). Groups 1, 3 and 4 included S. sonnei from Nepal and Thailand. Group 2 consisted of the $29 \mathrm{~S}$. sonnei isolates from Bhutan and 1 isolate from Nepal. S. sonnei isolates from Bhutan were closely related within the subgroup with $97-100 \%$ similarity. All 30 isolates in group 2 had a pattern of resistance to NA, CIP, SXT, $S$ and TE (Figure 2).
S. sonnei from Bhutan contained various plasmids with sizes ranging from $0.35 \mathrm{~Kb}$ to $98 \mathrm{~Kb}$. Nine isolates from Bhutan contained larger plasmids sized from $32 \mathrm{~Kb}$ to $98 \mathrm{~Kb}$ similar to 1 isolate from Nepal and 4 isolates from Thailand. Most isolates from all three sites contained variable plasmids sized from $0.35 \mathrm{~Kb}$ to $25 \mathrm{~Kb}$. At $62 \%$ similarity, dendrogram of the plasmid DNA patterns of $S$. sonnei was classified into 4 groups. Group 2 consisted of the $29 \mathrm{~S}$. sonnei isolates from Bhutan and 1 isolate from Nepal. Groups 1, 3 and 4 included S. sonnei isolates from Thailand and Nepal with variable antimicrobial susceptibility patterns [see Additional file 1].

\section{Replicon typing}

S. sonnei isolates were classified for plasmid incompatibility by PCR-based replicon typing (PBRT) with 21 primer pairs

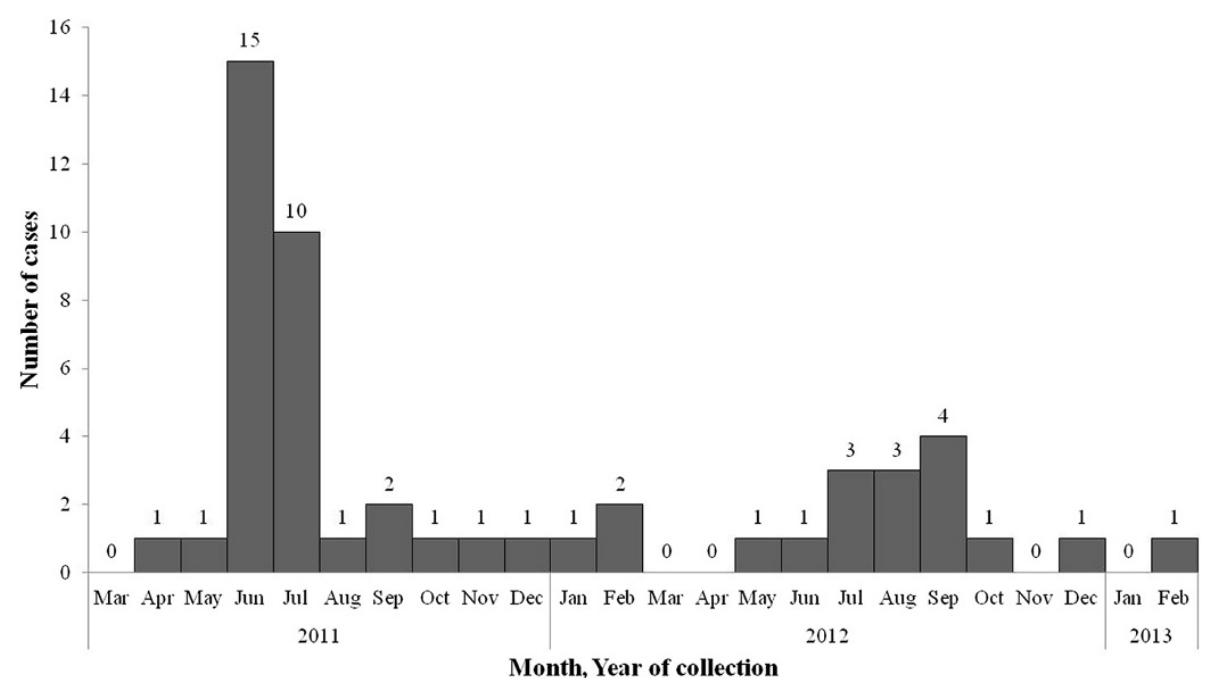

Figure 1 Epidemic curve of S. sonnei isolates in Thimphu, Bhutan from March 2011 to February 2013. 
Table 2 Characteristics of S. sonnei isolates from Bhutan, Nepal and Thailand

\begin{tabular}{|c|c|c|c|c|c|c|c|c|c|}
\hline \multirow{2}{*}{$\begin{array}{l}\text { Study } \\
\text { site }\end{array}$} & \multirow{2}{*}{$\begin{array}{l}\text { Number of } \\
\text { isolates }\end{array}$} & \multirow{2}{*}{$\begin{array}{c}\text { Resistance } \\
\text { pattern }\end{array}$} & \multicolumn{3}{|c|}{ MIC $(\mu \mathrm{g} / \mathrm{ml})$} & \multirow[t]{2}{*}{ Integrons } & \multicolumn{2}{|c|}{ DNA Gyrase A mutation } & \multirow{2}{*}{$\begin{array}{l}\text { Replicon } \\
\text { plasmid }\end{array}$} \\
\hline & & & AZM & NA & CIP & & Ser83 & Asp87 & \\
\hline Bhutan & 19 & S, SXT, TE, NA, CIP & $3.0-4.0$ & $>256$ & $3.0-4.0$ & Class 2 & Ser83Leu & Asp87Gly & ColE \\
\hline Bhutan & 9 & S, SXT, TE, NA, CIP & $3.0-4.0$ & $>256$ & $3.0-4.0$ & Class 2 & Ser83Leu & Asp87Gly & $\mathrm{B} / \mathrm{O}, \mathrm{ColE}$ \\
\hline Bhutan & 1 & S, SXT, TE, NA, CIP & 4.0 & $>256$ & 3.0 & Class 2 & Ser83Leu & Asp87Gly & I1, ColE \\
\hline Nepal & 1 & $\mathrm{~S}, \mathrm{SXT}, \mathrm{TE}, \mathrm{NA}, \mathrm{CIP}$ & 4.0 & $>256$ & 4.0 & Class 2 & Ser83Leu & Asp87Gly & ColE \\
\hline Nepal & 2 & $S, S X T, T E, N A$ & $3.0-4.0$ & $>256$ & $0.190-0.125$ & Class 2 & Ser83Leu & Neg & ColE \\
\hline Nepal & 1 & S & 3.0 & 3.0 & 0.008 & Neg & Neg & Neg & ColE \\
\hline Thailand & 1 & S, SXT, TE, NA, AM & 3.0 & $>256$ & 0.190 & Neg & Ser83Leu & Neg & $\mathrm{B} / \mathrm{O}, \mathrm{ColE}$ \\
\hline Thailand & 1 & $\mathrm{~S}, \mathrm{SXT}, \mathrm{TE}, \mathrm{AM}$ & 3.0 & 3.0 & 0.004 & Neg & Neg & Neg & I1, ColE \\
\hline Thailand & 2 & $\mathrm{~S}, \mathrm{SXT}, \mathrm{TE}, \mathrm{NA}, \mathrm{AM}$ & $3.0-4.0$ & 128 & 0.004 & Class 2 & Neg & Asp87Tyr & I1, ColE \\
\hline Thailand & 1 & S, SXT, TE, NA, & 4.0 & $>256$ & 0.004 & Class 2 & Ser83Leu & Neg & ColE \\
\hline
\end{tabular}

AZM, azithromycin; NA, nalidixic acid; CIP, ciprofloxacin; AM, ampicillin; SXT, trimethoprim-sulfamethoxazole; CRO, ceftriaxone; S, streptomycin; TE, tetracycline; Ser, Serine; Leu, Leucine; Asp, Aspartic acid; Gly, Glycine; Tyr, Tyrosine; Neg, negative result.

(B/O, FIC, A/C, P, T, K/B, W, FIIA, FIA, FIIB, Y, I1, Frep, $\mathrm{X}, \mathrm{HI} 1, \mathrm{~N}, \mathrm{HI} 2, \mathrm{~L} / \mathrm{M}$, ColE, IncR and IncU plasmid. All 38 isolates from Bhutan, Nepal, and Thailand carried the ColE plasmid. Nine isolates from Bhutan and one isolate from Thailand carried $\mathrm{B} / \mathrm{O}$ plasmid. One isolate from Bhutan and three isolates from Thailand carried the I1 plasmid (Table 2).

\section{PCR and sequencing analysis}

The gyrA genes of the $38 S$. sonnei isolates from Bhutan, Nepal and Thailand were amplified. The sequence analysis of the $470 \mathrm{bp}$ amplified products from $29 \mathrm{~S}$. sonnei isolates from Bhutan and one isolate from Nepal revealed the presence of two point mutations in the GyrA subunit (Ser83Leu and Asp87Gly) (Table 2). However, no amplified products were observed for the mphA gene in all $38 \mathrm{~S}$. sonnei isolates compared to an azithromycin resistant Enteroaggregative E. coli control.

All $38 \mathrm{~S}$. sonnei isolates had amplified products of class 1 integrons of approximately $700 \mathrm{bp}$. By DNA sequencing, the amplified products of 5 selected $S$. sonnei isolates from Bhutan showed no homologies to published gene cassettes of class 1 integrons but had 99\% identity of conserved hypothetical protein S. sonnei Ss046 in the NCBI database. Thus, S. sonnei in Bhutan did not contain resistance genes in class 1 integrons. The class 2 integrons of $38 \mathrm{~S}$. sonnei isolates were also amplified. The amplified products of 29 isolates from Bhutan and 3 isolates from Nepal were of similar size $(2.4 \mathrm{~Kb})$ but differed from the class 2 integrons in the 3 Thailand isolates $(\sim 3-4 \mathrm{~Kb})$. The DNA sequence alignment of class 2 integrons from 5 selected S. sonnei isolates from Bhutan had homology to dihydrofolate reductase (dfrA1), streptothricin acetyltransferase (sat1) and aminiglycoside adenyltransferase (aadA1) associated with resistance to trimethoprim, streptothricin and streptomycin/ spectinomycin, respectively. Moreover, RFLP of amplified products of the class 2 integrons in all 38 isolates with $\mathrm{XbaI}$, yielded the same DNA restricted-fragments of approximately $1,600 \mathrm{bp}$ and $800 \mathrm{bp}$ in $29 \mathrm{~S}$. sonnei isolates from Bhutan and 1 isolate from Nepal. The other Nepal and Thailand isolates had uncut DNA or undetectable bands.

\section{Discussion}

Over the last few years, MDR patterns in Shigella spp. have been reported in many countries [20]. In this study, all S. sonnei isolates from children in Thimphu, Bhutan in June and July, 2011 were resistant to multiple drugs like ciprofloxacin, nalidixic acid, trimethoprim-sulfamethoxazole, streptomycin and tetracycline. The MDR patterns of the S. sonnei from Bhutan differed from neighboring countries. In India, antimicrobial resistance in S. sonnei is more common than in other enteric bacteria with resistant to azithromycin, ciprofloxacin, furazolidone, nalidixic acid, norfloxacin, ofloxacin, trimethoprim-sulfamethoxazole, tetracycline [21]. In Bangladesh, most of S. sonnei isolates were resistant to nalidixic acid, sulfamethoxazoletrimethoprim, ampicillin, streptomycin and tetracycline [22]. In China, the antimicrobial resistance profiles of $S$. sonnei were nalidixic acid, piperacilline and ciprofloxacin [23]. In Nepal, S. sonnei isolates were resistant to azithromycin, ciprofloxacin, ceftriaxone, ampicillin, nalidixic acid and trimethoprim-sulfamethoxazole [24].

Shigellae usually harbor various plasmids, such as those required for bacterial invasion into the host intestinal epithelial cells and antibiotic resistance, which may range in number from two to as many as ten in one strain [25]. The PFGE and plasmid patterns of S. sonnei from Thimphu, Bhutan suggested clonality. S. sonnei in Thimphu, Bhutan carried the typical number of variable plasmid sizes from $0.35 \mathrm{~Kb}$ to $98 \mathrm{~Kb}$. These $S$. sonnei also contained the typical number of plasmids from seven to 
ten in one strain. The ability to recognize and categorize plasmids in homogeneous groups on the basis of their phylogenetic relatedness is helpful to analyze their distribution in nature and their relationship to host cells and to discover their evolutionary origins [26]. Classification of plasmids into incompatibility groups is desirable because specific plasmid types have been associated with virulence and/or antimicrobial resistance [15]. The plasmid replicon typing results showed that all S. sonnei isolates from Thimphu, Bhutan carried ColE plasmid as the same as control isolates from Nepal and Thailand. ColE plasmid which contains genes that code for colicin protein (bacteriocin protein) that can kill other bacteria and can replicate in the absence of de novo protein synthesis [26,27]. Furthermore, ColE plasmids co-resided with $\mathrm{B} / \mathrm{O}$ or $\mathrm{I} 1$ in several $S$. sonnei isolates. The simultaneous presence of the ColE plasmid with additional plasmids belonging to the $\mathrm{I} 1, \mathrm{~B} / \mathrm{O}$ or $\mathrm{A} / \mathrm{C}$ groups within the same parental strain suggested that the latter plasmids can participate in the mobilization of the ColE plasmids. B/O and I1 plasmid are plasmid incompatibility (Inc) groups capable of carrying transfer, MDR, and virulence functions [28].

Previous studies reported on the frequent incidence of class 2 integrons in Shigella isolates worldwide. DNA sequencing of the class 2 integrons in this study showed homologies with class 2 integrons but no homologies to published gene cassettes of class 1 integrons. In this study, 
S. sonnei from Thimphu, Bhutan carried three classic gene cassettes $(2,433 \mathrm{bp})$ in transposon $\mathrm{Tn} 7$ corresponding to previous results. S. sonnei isolates from Korea carried class 2 integrons $(2,224 \mathrm{bp})$ that consist of Tn7 with $d f r A 1$, sat 1 and aadA1 [29]. In Japan, two types of class 2 integrons in $S$. sonnei isolates were previously identified. One was the classical type (2,158 bp) with dfrA1, sat 1 and aadA1. The other type was shorter $(1,313 \mathrm{bp})$ and carried only two gene cassettes with $d f r A 1$ and sat1 [20]. In China, a 3,361 bp DNA fragment was found in S. sonnei with gene cassette arrays in this class 2 integrons of $d f r A 1$, sat1, aadA1 and orf X [17].

Nalidixic acid resistance among enteric bacteria is related to the presence of a single amino-acid substitution at either position 83 or position 87 of GyrA, while resistance to ciprofloxacin is related to the presence of at least one additional substitution in GyrA [30]. In this study, S. sonnei in Thimphu, Bhutan presented two mutations at position 83 (Serine to Leucine) and position 87 (Aspartic acid to Glycine) related to NA and CIP resistance. These two mutations conferred extremely high resistance to NA in S. sonnei in Bhutan that has been reported previously from $S$. sonnei isolates in Japan [31]. The expression of $m p h A$ gene is related to the decrease of AZM susceptibility [19]. In the USA, isolates with a susceptibility at $8 \mathrm{mg} / \mathrm{L}$ lacked $m p h A$ gene but isolates with higher MICs $(>64 \mathrm{mg} / \mathrm{L})$ to AZM reportedly contained a plasmid-encoded $m p h A$ gene [19]. In this study, MIC range of AZM was $3.0-4.0 \mu \mathrm{g} / \mathrm{ml}$ and PCR results showed that mphA gene was not present.

\section{Conclusions}

This study described the recent antimicrobial resistance among S. sonnei strains isolated from Thimphu, Bhutan in June and July, 2011 and molecular characterization of these S. sonnei. Plasmid patterns, PFGE and uniform MDR suggest that these $29 \mathrm{~S}$. sonnei isolates are closely related. All S. sonnei isolates carried common ColE plasmid that co-resided with $\mathrm{B} / \mathrm{O}$ or $\mathrm{I} 1$ plasmid in many isolates. Further study and comparison with other isolates from the region may provide information on the development and continued spread of antibiotic resistance in S. sonnei.

\section{Additional file}

\section{Additional file 1: The dendrogram of plasmid profile. The} dendrogram obtained by cluster analysis of the plasmid patterns of $S$. sonnei from Bhutan, Nepal and Thailand combined with antibiotic susceptibility.

\section{Competing interests}

The authors declare that they have no competing interests.

\section{Authors' contributions}

SR performed laboratory assay on molecular typing, data analysis and prepared manuscript. TD coordinated laboratory work between Bhutan and AFRIMS, and performed laboratory work in Bhutan. PP and PN performed laboratory assay on molecular typing and data analysis. OS provided comments and suggestions on molecular methods and reviewed the manuscript. KP performed laboratory on antimicrobial susceptibility testing and data analysis. KPT provided stool samples from Jigme Dorji Wangchuk National Referral Hospital to the Public Health Laboratory in Bhutan. SW and LB contributed to study design and protocol development, and coordinated laboratory infrastructure development and quality control programs in the Public Health Laboratory. CJM oversaw any laboratory work done in the Department of Enteric Diseases at AFRIMS and reviewed the manuscript. All authors' read and approved the final manuscript.

\section{Acknowledgments}

We are grateful to the staff of Public Health Laboratory and Jigme Dorji Wangchuk National Referral Hospital, Thimphu, Bhutan for kindly providing stool samples in this study. We also thank to Alessandra Carottoli, Parasitic and Immune-mediated Diseases, Rome, Italy for kindly provided reference plasmid controls.

This study was supported by grants from the Armed Forces Health Surveillance Center-Global Emerging Infections System (AFHSC-GEIS), Silver Spring, MD, U.S.A.

\section{Disclaimer for research not involving animals}

The views expressed in this article are those of the author(s) and do not reflect the official policy of the Department of the Army, Department of Defense, or the U.S. Government.

\section{Author details}

'Department of Enteric Diseases, Armed Forces Research Institute of Medical Sciences (AFRIMS), Bangkok, Thailand. ²Public Health Laboratory, Department of Public Health, Ministry of Health, Royal Government of Bhutan, Thimphu, Bhutan. ${ }^{3}$ Department of Pediatrics, Jigme Dorji Wangchuk National Referral Hospital, Kawa Jangsa, Thimphu, Bhutan.

Received: 2 August 2013 Accepted: 14 February 2014 Published: 20 February 2014

\section{References}

1. World Health Organization: Fact sheets: Diarrhoeal disease 2013. [http://www.who.int/mediacentre/factsheets/fs330/en/index.html].

2. Royal Government of Bhutan: Dzongkha Population Projections 2006-2015: National Statistics Bureau. [http://www.nsb.gov.bt/publication/ files/pub3uu3600pb.pdf]

3. Ministry of Health: Annual Health Bulletin 2009. [http://www.health.gov.bt/ ahb2009.php]

4. Ministry of Health: Annual Health Bulletin 2011. [http://www.health.gov.bt/ ahb2011.php]

5. Darnal JB, Nepal HK, Damchu, Wangchuk LZ, Doung-ngern P, Swaddiwudhipong W: An Outbreak of Shigellosis in a Remote Village of Mongar District, Bhutan from March to April 2011. OSIR 2012, 5(2):1-8.

6. Rowe-Magnus DA, Guerout AM, Mazel D: Bacterial resistance evolution by recruitment of super-integron gene cassettes. Mol Microbiol 2002, 43(6):1657-1669.

7. Gravel A, Fournier B, Roy PH: DNA complexes obtained with the integron integrase Intl1 at the attl1 site. Nucleic Acids Res 1998, 26(19):4347-4355.

8. White PA, Mclver CJ, Rawlinson WD: Integrons and gene cassettes in the enterobacteriaceae. Antimicrob Agents Chemother 2001, 45(9):2658-2661.

9. DeLappe N, O'Halloran F, Fanning S, Corbett-Feeney G, Cheasty T, Cormican M: Antimicrobial resistance and genetic diversity of Shigella sonnei isolates from western Ireland, an area of low incidence of infection. J Clin Microbiol 2003, 41(5):1919-1924.

10. DeNap JC, Hergenrother PJ: Bacterial death comes full circle: targeting plasmid replication in drug-resistant bacteria. Org Biomol Chem 2005, 3(6):959-966.

11. Podzorski RP, Persing DH: Molecular detection and identification of microorganisms. In Manual of Clinical Microbiology. 6th edition. Edited by Murray PR, Baron EJ, Pfaller MA, Tenover FC, Yolken RH. Washington DC: ASM Press; 1995:130-157. 
12. Clinical and Laboratory Standards Institute: Performance Standards for Antimicrobial Susceptibility Testing; Twenty-First Informational Supplement M100S21. Wayne, Pennsylvannia: Clinical and Laboratory Standards Institute; 2011.

13. Centers for Disease Control and Prevention: One-Day (24-28 h) Standardized Laboratory Protocol for Molecular Subtyping of Escherichia coli O157:H7, Salmonella serotypes, Shigella sonnei, and Shigella flexneri by Pulsed Field Gel Electrophoresis (PFGE). California: Centers for Disease Control and Prevention; 2009.

14. Birnboim HC, Doly J: A rapid alkaline extraction procedure for screening recombinant plasmid DNA. Nucleic Acids Res 1979, 7(6):1513-1523.

15. Johnson TJ, Wannemuehler YM, Johnson SJ, Logue CM, White DG, Doetkott C, Nolan LK: Plasmid replicon typing of commensal and pathogenic Escherichia coli isolates. Appl Environ Microbiol 2007, 73(6):1976-1983.

16. Garcia-Fernandez A, Fortini D, Veldman K, Mevius D, Carattoli A: Characterization of plasmids harbouring qnrS1, qnrB2 and qnrB19 genes in Salmonella. J Antimicrob Chemother 2009, 63(2):274-281.

17. Pan JC, Ye R, Meng DM, Zhang W, Wang HQ, Liu KZ: Molecular characteristics of class 1 and class 2 integrons and their relationships to antibiotic resistance in clinical isolates of Shigella sonnei and Shigella flexneri. J Antimicrob Chemother 2006, 58(2):288-296.

18. Giraud E, Brisabois A, Martel JL, Chaslus-Dancla E: Comparative studies of mutations in animal isolates and experimental in vitro- and in vivo-selected mutants of Salmonella spp. suggest a counterselection of highly fluoroquinolone-resistant strains in the field. Antimicrob Agents Chemother 1999, 43(9):2131-2137.

19. Howie RL, Folster JP, Bowen A, Barzilay EJ, Whichard JM: Reduced azithromycin susceptibility in Shigella sonnei, United States. Microb Drug Resist 2010, 16(4):245-248.

20. Ahmed AM, Furuta K, Shimomura K, Kasama Y, Shimamoto T: Genetic characterization of multidrug resistance in Shigella spp. from Japan. J Med Microbiol 2006, 55(Pt 12):1685-1691.

21. Ghosh S, Pazhani GP, Chowdhury G, Guin S, Dutta S, Rajendran K, Bhattacharya MK, Takeda Y, Niyogi SK, Nair GB, et al: Genetic characteristics and changing antimicrobial resistance among Shigella spp. isolated from hospitalized diarrhoeal patients in Kolkata, India. J Med Microbiol 2011, 60(Pt 10):1460-1466.

22. Talukder KA, Islam Z, Dutta DK, Islam MA, Khajanchi BK, Azmi IJ, Iqbal MS, Hossain MA, Faruque AS, Nair GB, et al: Antibiotic resistance and genetic diversity of Shigella sonnei isolated from patients with diarrhoea between 1999 and 2003 in Bangladesh. J Med Microbiol 2006, 55(Pt 9):1257-1263.

23. Mehata S, Duan GC: Molecular mechanism of multi-drug resistance in Shigella isolates from rural China. Nepal Med Coll J 2011, 13(1):27-29.

24. Surdeanu M, Pencu E, Tonciu M, Mihai I, Ciudin L: Differentiation of Shigella strains by plasmid profile analysis, serotyping and phage typing. Roum Arch Microbiol Immunol 2000, 59(1-2):103-117.

25. Haider K, Huq MI, Talukder KA, Ahmad QS: Electropherotyping of plasmid DNA of different serotypes of Shigella flexneri isolated in Bangladesh. Epidemiol Infect 1989, 102(3):421-428.

26. Carattoli A: Resistance plasmid families in Enterobacteriaceae. Antimicrob Agents Chemother 2009, 53(6):2227-2238.

27. Riley MA, Gordon DM: A survey of Col plasmids in natural isolates of Escherichia coli and an investigation into the stability of Col-plasmid lineages. J Gen Microbiol 1992, 138(7):1345-1352.

28. Johnson TJ, Nolan LK: Pathogenomics of the virulence plasmids of Escherichia coli. Microbiol Mol Biol Rev 2009, 73(4):750-774

29. Seol SY, Kim YT, Jeong YS, Oh JY, Kang HY, Moon DC, Kim J, Lee YC, Cho DT, Lee JC: Molecular characterization of antimicrobial resistance in Shigella sonnei isolates in Korea. J Med Microbiol 2006, 55(7):871-877.

30. Ruiz J: Mechanisms of resistance to quinolones: target alterations, decreased accumulation and DNA gyrase protection. $J$ Antimicrob Chemother 2003, 51(5):1109-1117.

31. Hirose K, Terajima J, Izumiya H, Tamura K, Arakawa E, Takai N, Watanabe H: Antimicrobial susceptibility of Shigella sonnei isolates in Japan and molecular analysis of $\mathrm{S}$. sonnei isolates with reduced susceptibility to fluoroquinolones. Antimicrob Agents Chemother 2005, 49(3):1203-1205.

doi:10.1186/1756-0500-7-95

Cite this article as: Ruekit et al:: Molecular characterization and PCR-based replicon typing of multidrug resistant Shigella sonnei isolates from an outbreak in Thimphu, Bhutan. BMC Research Notes 2014 7:95. 